\title{
Asymmetric radiation-induced toroidal flow and improved confinement in tokamaks
}

\author{
R. Singh and P. K. Kaw \\ Institute for Plasma Research, Bhat, Gandhinager-382 428, India
}

\author{
A. L. Rogister \\ Institut für Plasmaphysik, Forschungszentrum Jülich GmbH, EURATOM-FZJ Association, \\ Juelich, D-52425, Germany \\ V. Tangri \\ Institute for Plasma Research, Bhat, Gandhinager-382 428, India
}

(Received 14 November 2005; accepted 2 March 2006; published online 18 April 2006)

\begin{abstract}
The role of impurity radiation in influencing the toroidal flow and radial electric fields (parameters critical for determining turbulent transport) has been studied on the edge of a tokamak plasma. It is demonstrated for the first time that the impurities distributed in an asymmetric (poloidally) manner may lead to significant density and temperature perturbations on magnetic surfaces. These, in turn, interact with the $\theta$ dependent toroidal field variations and yield a mean divergence of the stress tensor driving strong neoclassical toroidal flows. A self-consistent theory of interplay of equilibrium, fluctuations, neoclassical flows, and $\vec{E} \times \vec{B}$ shear rotation in a tokamak is also presented. It is shown that the resulting enhanced toroidal velocity shear on the outer radiative layers produces a stabilizing effect on the well known instabilities (which determine edge transport) such as the drift resistive ballooning mode, the drift trapped electron mode, and the ion temperature gradient mode. For various values of the radiation asymmetry parameter, investigation of the turbulent particle flux as a function of the density gradient shows that the plasma can undergo a bifurcation into a better-confined state with a peaked density. (C) 2006 American Institute of Physics.
\end{abstract}

[DOI: 10.1063/1.2192509]

\section{INTRODUCTION}

It is now widely recognized that edge physics plays a key role in determining the global confinement performance of tokamak discharges. Several experiments have demonstrated that the transition from L-mode (low confinement) to $\mathrm{H}$-mode (high confinement) can be affected by plasma flows in the edge of a tokamak plasma. A great deal of work ${ }^{1}$ has been done in recent years on the origin of toroidal flows and radial electric fields in the tokamak edge plasma. There is evidence $^{2}$ that flows with strong shear are induced in the plasma even in the absence of any net toroidal momentum injection into the device. This has led to suggestions that the flows may be associated with neoclassical ambipolar transport effects, ${ }^{1}$ polarization of the edge plasma by ion loss and radio-frequency-induced ion compression effects, ${ }^{3}$ effects associated with asymmetric neutral gas injection, ${ }^{4}$ etc. In this paper we explore the idea that poloidally asymmetric radiation from the tokamak edge plasmas may be responsible for radial electric fields and toroidal flows. We take our cue from the physics of the radiative improved (RI) confinement mode, which is triggered under certain conditions in limiter tokamaks when seeding impurities like neon or argon are introduced. This new operational regime was discovered in the Impurity Study Experiment (ISX-B) tokamak ${ }^{5}$ and then confirmed and thoroughly reinvestigated on the Tokamak Experiment for Technology Oriented Research (TEXTOR-94). ${ }^{6,7}$ Compared to the L (low) confinement mode, the RI mode is characterized by more peaked density and temperature profiles, larger operating densities $\left(\bar{N}_{e}\right.$ may be well above the Greenwald limit), and larger confinement times $\left[\tau_{E}\right.$ may be close to edge localized mode (ELM) $\mathrm{H}$-mode values]. The mechanisms considered presently to lead to confinement improvement in the RI mode are largely based on the reduction of the growth rate of the toroidal ion temperature gradient (ITG) mode when the plasma $Z_{\text {eff }}$ increases. ${ }^{8}$ It is argued that the plasma density profile peaks when the ITG mode gets suppressed owing to the pinching effects associated with the dissipative-trapped electron (DTE) mode. ${ }^{9}$ The density peaking further suppresses the ITG and the plasma undergoes a bifurcation into the improved confinement, high-density regime. However, significantly there is experimental evidence ${ }^{10}$ that the toroidal flow velocity in the impurity seeded plasma is larger and more peaked than in the nonseeded plasma (i.e., has more $\vec{E} \times \vec{B}$ velocity shear); yet there has been little effort to explain this feature or to make use of it to contribute to the stabilization of the ITG mode and other edge instabilities. In all likelihood, both effects are taking place in RI mode discharges, namely a direct reduction of the ITG growth rate due to the increase of $Z_{\text {eff }}$ and a suppression of the turbulence due to the increased velocity shear. It is therefore of significant interest to examine novel mechanisms associated with impurity injection which contribute to the $\vec{E} \times \vec{B}$ velocity shear profile.

This paper explores one such mechanism. The basic physics of the generation of neoclassical toroidal and poloidal rotation velocities in high collisionality plasmas goes as 
follows. When an impurity such as neon is injected into a typical L-mode discharge, it radiates copiously until it reaches lithium- and beryllium-like states. This occurs typically in the outer $20 \%-25 \%$ of the discharge minor radius in relatively small devices. Furthermore, the impurities are not distributed symmetrically in the poloidal direction but show in-out and up-down asymmetries; ${ }^{7}$ quantitatively, the asymmetry in concentration could be as much as a factor of 2 . This concentration asymmetry leads to large poloidal asymmetries in the radiation from even thermally stable plasmas. Asymmetric radiation generates temperature, density, potential, and parallel ion flow perturbations on the magnetic surface that interact with the theta-dependent toroidal field variations to produce a mean divergence of the stress tensor. According to neoclassical theory ${ }^{11}$ such a mean divergence drives significant mean toroidal flows on these surfaces. We note in passing that this effect will be weaker in bigger, hotter plasmas (like those in a fusion reactor) because the large parallel electron thermal conduction will prevent the formation of significant electron temperature perturbations on a magnetic surface. Since the basic process is at work in the radiative region only, a significantly enhanced toroidal velocity shear $U_{\phi, i}^{\prime}$ (where the prime denotes differentiation with respect to $r$ ) is produced in and near the radiation layer. This, in turn, increases the radial electric field imposed by the radial force balance equation $E_{r} / B_{\phi}=\left(T_{i} / e B_{\phi}\right) \partial \ln P_{i} / \partial r$ $+\left(B_{\theta} / B_{\phi}\right) U_{\phi i}-U_{\theta i}$ and the $\vec{E} \times \vec{B}$ rotation velocity shear $\left\langle E_{r} / B_{\phi}\right\rangle^{\prime}=\left\langle U_{E}\right\rangle^{\prime}=\left\langle U_{\theta, i}\right\rangle^{\prime}-(\varepsilon / q)\left\langle U_{\phi, i}\right\rangle^{\prime}-4\left(B / B_{\phi}\right) \varepsilon_{i}^{2} a_{i} c_{i} R^{-2} p(1$ $-p)$. [Here, we have introduced the notations for thermal velocity, Larmor radius, and cyclotron frequency of ions: $c_{i}=\sqrt{T_{i} / m_{i}}, a_{i}=c_{i} / \Omega_{i}$, and $\Omega_{i}=e B / m_{i}$. Other notations introduced are those of the peaking factor: $p=L_{T_{i}} / L_{N}=\eta_{i}^{-1}$; the temperature and density gradient scales: $L_{T}$ and $L_{N}$; the flux surface average notation \langle\rangle$, \varepsilon_{i}=R / 2 L_{T_{i}}$, , the major radius of the discharge, and $q=r B_{\phi} / R B_{\theta}$, the safety factor. Furthermore, $U_{\theta, i} \approx-1.83\left(T_{i} / e B_{\phi}\right)\left(\partial \ln T_{i} / \partial r\right)$ is the poloidal ion velocity in Pfirsh-Schlüter regime. ${ }^{11}$ ] Following recent theoretical work, ${ }^{12}$ we expect the radiation asymmetry driven $\vec{E}$ $\times \vec{B}$ shear flow to suppress the most important instabilities in the radiative edge, namely the ITG,$^{13}$ DTE, ${ }^{8}$ and high- $m$ drift resistive ballooning (DRB) ${ }^{14}$ modes. This leads to a reduction in particle and thermal convective fluxes that sharpens the density and temperature profiles locally. If this sharpening leads to further suppression of the turbulence, then the plasma may bifurcate into a better-confined state.

The remainder of the paper is organized as follows. The basic equations and coordinate system used are described in Sec. II. In Sec. III, the general formulation of neoclassical toroidal flow and ordering of various terms are given. In Sec. IV, we discuss the applications of our theory to experiments. A brief summary of our results is given in Sec. V.

\section{BASIC EQUATION}

We start with Braginskii's two-fluid equations ${ }^{15}$ including the Mikhailovskii and Tsypin corrections to the stress tensor term in the ion momentum equation. ${ }^{16}$ These equations are the ion continuity equation, the ion and electron momentum equation, the energy equations for ion and electron species, the parallel electron momentum equation,

$$
\begin{aligned}
& \frac{\partial N_{i}}{\partial t}+\vec{\nabla} \cdot N_{i} \vec{U}_{i}=0, \\
& m_{j} N_{j}\left(\frac{\partial}{\partial t}+\vec{U}_{j} \cdot \vec{\nabla}\right) \vec{U}_{j} \\
& \quad=-\vec{\nabla} P_{j}-\vec{\nabla} \cdot \vec{\pi}_{j}+e_{j} N_{j}\left(\vec{E}+\vec{U}_{j} \times \vec{B}\right), \\
& \frac{3}{2} N_{i}\left(\frac{\partial}{\partial t}+\vec{U}_{i} \cdot \vec{\nabla}\right) T_{i}+P_{i} \vec{\nabla} \cdot \vec{U}_{i}=-\vec{\nabla} \cdot \vec{q}_{i}, \\
& \frac{3}{2} N_{e}\left(\frac{\partial}{\partial t}+\vec{U}_{e} \cdot \vec{\nabla}\right) T_{e}+P_{e} \vec{\nabla} \cdot \vec{U}_{e}=-\vec{\nabla} \cdot \vec{q}_{e}-Q_{\mathrm{rad}}(\chi),
\end{aligned}
$$

where $j=i, e$, the charge $e_{j}=e$ for the ion and $e_{j}=-e$ for the electron, $\vec{\pi}_{i}$ is the ion stress tensor given by Braginskii ${ }^{15}$ and Mikhailovskii and Tsypin ${ }^{16}$ (see the Appendix), $\nu_{\mathrm{cx}}$ is the charge exchange rate, $Q_{\text {rad }}(\chi)$ is the radiation power density loss due to impurities radiation, $\vec{q}_{i}=-\kappa_{\| i} \nabla_{\|} T_{i}-\kappa_{\perp i} \nabla_{\perp} T_{i}$ $+\kappa_{x i} \hat{n} \times \vec{\nabla} T_{i}$ and $\vec{q}_{e}=-\kappa_{\| e} \nabla_{\|} T_{e}-\kappa_{\perp e} \nabla_{\perp} T_{e}+\kappa_{x e} \hat{n} \times \nabla T_{e}$ are the ion and electron diffusive thermal fluxes, $\kappa_{\| i}$ $=3.9\left(N_{i} T_{i} / m_{i} \nu_{i}\right)$ and $\kappa_{\| e}=3.2\left(N_{e} T_{e} / m_{e} \nu_{e}\right)$ are the parallel ion and electron diffusion coefficients, $\kappa_{\perp i}$ and $\kappa_{\perp e}$ are the perpendicular ion and electron diffusion coefficients, $\kappa_{x i}$ $=5 N_{i} T_{i} / 2 m_{i} \Omega_{i}$ and $\kappa_{x e}=5 N_{e} T_{e} / 2 m_{e} \Omega_{e}$ are the cross field diffusion coefficients (diamagnetic heat flux) associated with ions and electrons; other plasma notations are standard. Note we have neglected the electron energy transferred through the equilibration rate process since $1 \gg\left(\lambda_{e}^{\mathrm{mfp}} / q R\right)^{2}>m_{e} / m_{i}$ is taken in the analysis (where $\lambda_{e}^{\mathrm{mfp}}$ is the electron mean free path).

We use the coordinate system $(\hat{p}, \hat{b}, \hat{n})$ that is tied to magnetic field, $\hat{n}=\vec{B} / B$, the unit vector along the magnetic field lines, $\hat{p}$ is orthogonal to the magnetic surface, and $\hat{b}=\hat{n} \times \hat{p}$ is the binormal component. The unit vectors $\hat{p}, \hat{b}, \hat{n}$ are related to the flux coordinates $\hat{e}_{\psi}, \hat{e}_{\chi}, \hat{e}_{\phi}$ by

$$
\begin{aligned}
& \hat{p}=\hat{e}_{\psi}, \quad \hat{b}=\left(\frac{B_{\phi}}{B}\right) \hat{e}_{\chi}-\left(\frac{B_{\chi}}{B}\right) \hat{e}_{\phi}, \\
& \hat{n}=\left(\frac{B_{\chi}}{B}\right) \hat{e}_{\chi}+\left(\frac{B_{\phi}}{B}\right) \hat{e}_{\phi} .
\end{aligned}
$$

The differential operators can be written as

$$
\begin{aligned}
\hat{p} \cdot \vec{\nabla} & \equiv h_{\psi}^{-1} \frac{\partial}{\partial \psi} \sim \frac{\partial}{\partial r} \sim L_{\psi}^{-1}, \\
\hat{b} \cdot \vec{\nabla} & \equiv\left(\frac{B_{\phi}}{B}\right) h_{\chi}^{-1} \frac{\partial}{\partial \chi} \sim \frac{1}{r} \frac{\partial}{\partial \theta} \sim r^{-1}, \\
\hat{n} \cdot \vec{\nabla} & \equiv\left(\frac{B_{\chi}}{B}\right) h_{\chi}^{-1} \frac{\partial}{\partial \chi} \sim \frac{1}{q R} \frac{\partial}{\partial \theta} \sim(q R)^{-1},
\end{aligned}
$$

where $h_{\psi}=1 / h_{\phi} B_{\chi}, h_{\chi}=J B_{\chi}, J=h_{\psi} h_{\chi} h_{\phi}$ is the Jacobian of the transformation, $\psi$ is the poloidal magnetic flux, $\chi$ is the gen- 
eralized poloidal angle, $\phi$ is the toroidal angle, $\nu$ $=h_{\chi} B_{\phi} / h_{\phi} B_{\chi} \sim r B_{\phi} / R B_{\chi}$ is the pitch of the field lines, and $q=\oint \nu d \chi$ is the safety factor. We note that $h_{\phi}=R_{0}(1$ $+\varepsilon \cos \chi)$ and $B_{\phi}=B_{\phi 0}(1-\varepsilon \cos \chi)$ in case of a large aspect ratio tokamak with a circular cross section, where $\varepsilon=r / R_{0}$ is the inverse aspect ratio, $r$, and respectively $R_{0}$ the minor and major radii. In this paper we use the notations and ordering as given in the paper by Rogister ${ }^{17}$ unless otherwise mentioned. We note that these notations can easily be identified with the standard tokamak coordinates system $[\psi-\operatorname{radial}(r), \chi$-poloidal $,(\theta), \phi$-toroidal $(\phi)]$ in the limit of a large aspect ratio tokamak with a circular cross section and toroidal symmetry $(\partial / \partial \phi \equiv 0)$.

\section{NEOCLASSICAL TOROIDAL FLOW}

We now present the basic model calculation demonstrating these effects. We first examine the toroidal flow, which may be driven due to poloidally asymmetric radiation from tokamak plasma. The scaling relevant to the edge is defined as $^{17}$

$$
\begin{aligned}
\frac{r}{q R} & \sim \frac{c_{i}}{q R \nu_{i}} \sim \frac{L_{\psi}}{r} \sim\left(\frac{m_{e}}{m_{i}}\right)^{1 / 4} \sim\left(\frac{a_{i}}{L_{\psi}}\right)^{1 / 2} \sim \mu \ll 1 \sim \frac{L_{n}}{L_{\psi}} \\
& \sim \frac{L_{T}}{L_{\psi}} .
\end{aligned}
$$

We order the ion flow velocities with respect to the ion thermal velocity as follows: ${ }^{17}$

$$
U_{\phi i} \sim \mu c_{i}, \quad U_{\chi^{i}} \sim \mu^{2} c_{i}, \quad U_{\psi i} \approx 0,
$$

where $\nu_{i}$ is the ion collision frequency $\left[=10^{-12}\right.$ $\times Z_{\text {eff }} N_{i} / T_{i}^{3 / 2}\left(2 A_{i}\right)^{1 / 2}$ if $N_{i}$ is expressed in $\mathrm{m}^{-3}$ and $T_{i}$ in $\mathrm{eV}$; $A_{i}$ is the ion atomic mass], $m_{i}\left(m_{e}\right)$ the ion (electron) mass, and $\mu$ a small parameter that we use later for expanding the basic equations and calculating the neoclassical toroidal flow.

Summing up the projection of ion and electron momentum equations onto toroidal direction, we obtain

$$
J_{\psi}=\frac{1}{B_{\chi}} \hat{e}_{\phi} \cdot\left[\vec{\nabla} \cdot \vec{\pi}_{i}+m_{i} N_{i}\left(\frac{\partial}{\partial t}+\vec{U}_{i} \cdot \vec{\nabla}\right) \vec{U}_{i}\right] .
$$

The convective term in the above momentum Eq. (8) can be expressed as

$$
\begin{aligned}
N_{i} \hat{e}_{\phi} \cdot\left[\left(\vec{U}_{i} \cdot \vec{\nabla}\right) \vec{U}_{i}\right] \\
=-J^{-1} U_{\phi i}\left(\frac{\partial}{\partial \psi}\left(h_{\chi} h_{\phi} N_{i} U_{\psi i}\right)+\frac{\partial}{\partial \chi}\left(h_{\psi} h_{\phi} N_{i} U_{\chi i}\right)\right) \\
+\left(J h_{\phi}\right)^{-1}\left(\frac{\partial}{\partial \psi}\left(h_{\chi} h_{\phi}^{2} U_{\psi i} U_{\phi i}\right)+\frac{\partial}{\partial \chi}\left(h_{\psi} h_{\phi}^{2} U_{\chi i} U_{\phi i}\right)\right) .
\end{aligned}
$$

Here we have made use of a tensorial relation $\vec{\nabla} \hat{e}_{\phi}=-\hat{e}_{\phi} \vec{\nabla} \ln h_{\phi}$.

In a general coordinate system, the continuity Eq. (1) is given as

$$
\frac{\partial N_{i}}{\partial t}+J^{-1}\left(\frac{\partial}{\partial \psi}\left(h_{\chi} h_{\phi} N_{i} U_{\psi}\right)+\frac{\partial}{\partial \chi}\left(h_{\psi} h_{\phi} N_{i} U_{i \chi}\right)\right)=0 .
$$

Combining Eqs. (8)-(10) and integrating the resulting expression over magnetic flux surfaces leads to the relation

$$
\begin{aligned}
& \oint J h_{\phi} \frac{d \chi}{2 \pi} \frac{\partial\left(m_{i} N_{i} U_{\phi}\right)}{\partial t}+\oint \hat{e}_{\phi} \cdot\left(\vec{\nabla} \cdot \vec{\pi}_{i}\right) J h_{\phi} \frac{d \chi}{2 \pi} \\
& +\frac{\partial}{\partial \psi} \oint J h_{\phi} h_{\psi}^{-1} m_{i} N_{i} U_{\psi i} U_{\phi i} \frac{d \chi}{2 \pi}=\oint J_{\psi} h_{\chi} h_{\phi} \frac{d \chi}{2 \pi},
\end{aligned}
$$

where the third term on the right-hand side (RHS) of Eq. (11) corresponds to the radial angular momentum flux.

In the absence of neoclassical radial convection effects and radial current, Eq. (11) can be rewritten as

$$
\begin{aligned}
\oint J h_{\phi} \hat{e}_{\phi} \cdot(\vec{\nabla} \cdot \overleftrightarrow{\pi}) d \chi=\frac{\partial}{\partial \psi} \oint J h_{\phi} h_{\chi}^{-1} \pi_{\psi \phi} d \chi=0, \\
0=\oint \hat{e}_{\phi} \cdot\left(\vec{\nabla} \cdot \vec{\pi}_{i}\right) J h_{\phi} d \chi \\
=\frac{\partial}{\partial \psi} \oint h_{\psi}^{-1} J h_{\phi} d \chi\left(\pi_{3-4, i}\right)_{\psi \phi} \\
\quad+\frac{\partial}{\partial \psi} \oint h_{\psi}^{-1} J h_{\phi} d \chi\left[\left(\pi_{1-2, i}\right)_{\psi \phi}\right] \\
=-\frac{m_{i}}{e} \frac{\partial}{\partial \psi}\left[h_{\phi}^{2} B_{\phi}^{2} \oint d \chi\left(\frac{P_{i}}{B^{4}} \frac{\partial}{\partial \chi}\left(U_{\| i} B\right)+\frac{8}{5} \frac{q_{\| i}}{B^{4}} \frac{\partial B}{\partial \chi}\right)\right] \\
\quad-\frac{12}{10} \frac{m_{i}}{e} \frac{\partial}{\partial \psi}\left[h_{\phi}^{2} B_{\phi}^{2} \oint d \chi\left(\frac{\nu_{i}}{\Omega_{i}} \frac{B_{\chi} J}{B_{\phi}} \frac{P_{i}}{B^{2}} h_{\psi}^{-1} \frac{\partial U_{\| i}}{\partial \psi}\right)\right] .
\end{aligned}
$$

We expand the equilibrium quantities in powers of the inverse aspect ratio (i.e., $\varepsilon \sim \mu$ ) and seek solutions of the form

$$
\begin{aligned}
& F(\psi, \chi)=F^{(0)}(\psi)\left[1+\mu f^{(1)}(\psi, \chi)+O\left(\mu^{2}\right)\right], \\
& B / B_{0}=(1-\varepsilon \cos \chi), \quad b^{(1)}(\psi, \chi) \equiv-\varepsilon \cos \chi,
\end{aligned}
$$

where $F(\psi)=N_{i}(\psi), T_{i}^{(0)}(\psi), \Phi(\psi), \vec{U}_{i, e}(\psi)$, or $B(\psi)$, and the equilibrium variables are functions of $\psi$ the poloidal magnetic flux alone, whereas $f(\psi, \chi)=n_{i}(\psi, \chi), t_{i}(\psi, \chi), \phi(\psi, \chi)$, $\vec{u}_{i, e}(\psi, \chi)$, or $b(\psi, \chi)$ are the perturbed variables that are functions of $\psi$ and $\chi$, the generalized poloidal angle.

To bring the asymmetric radiation effects in generation of neoclassical toroidal flow, we model the impurities power radiation as a function of poloidal coordinate as

$$
Q_{\mathrm{rad}}=Q_{\mathrm{rad}}^{(0)}\left[1+\Delta_{1} \cos \left(\chi-\chi^{*}\right)\right] \equiv Q_{\mathrm{rad}}\left[1+g\left(\chi, \chi^{*}\right)\right],
$$

where $Q_{\mathrm{rad}}^{(0)}$ is the averaged radiation power density, where $\chi$ is the poloidal angle, $\chi=0$ corresponds to the low field side midplane, $\chi^{*}$ to the position of maximum radiation, and $\Delta_{1}$ to the degree of asymmetry.

Substituting Eq. (14) into Eq. (13), we get 


$$
\begin{gathered}
\oint\left[\left(n^{(1)}+t_{i}^{(1)}-4 b^{(1)}\right)\left(\frac{\partial u_{\| i}^{(1)}}{\partial \chi}+U_{\| i}^{(0)} \frac{\partial b^{(1)}}{\partial \chi}\right)\right. \\
\left.-1.6 \chi_{\| i}^{(0)} \frac{B_{\chi}^{(0)}}{B_{0}} \frac{1}{h_{\chi}^{(0)}}\left(\frac{\partial t_{i}^{(1)}}{\partial \chi} \frac{\partial b^{(1)}}{\partial \chi}\right)\right] \frac{d \chi}{2 \pi} \\
+\frac{12}{10} \frac{P_{i}^{(0)}}{B_{0}^{2}} \frac{\nu_{i}^{(0)}}{\Omega_{i}^{(0)}} \frac{h_{\chi}^{(0)}}{B_{\phi 0}} \frac{1}{h_{\psi}^{(0)}} \frac{\partial U_{\| i}^{(0)}}{\partial \psi}=0,
\end{gathered}
$$

where $p_{j}^{(1)}=p_{j} / P_{j}^{(0)}, n_{j}^{(1)}=n_{j} / N_{j}^{(0)}, t_{j}^{(1)}=t_{j} / T_{j}^{(0)}, \phi^{(1)}=e \phi / T_{i}^{(0)}$ are the normalized perturbed variables, respectively, and $u_{\| i}^{(1)}$ is the perturbed parallel ion velocity, $\chi_{\| i}=3.9 T_{i}^{(0)} / m_{i} \nu_{i}^{(0)}$, $\nu_{i}^{(0)}$ is the ion collision frequency, and $j=i, e$.

To evaluate the relation between ion temperature, potential, and density perturbations, we first write the electron temperature perturbation from electron energy equation. In the limit $\left(\Omega_{e} / \nu_{e}\right)\left(r L_{T} / q^{2} R^{2}\right)>1$ [where parallel electron thermal conduction $\propto T_{e}^{e} / m_{e} \nu_{e} q^{2} R^{2}$ dominates over convection processes $\left.\propto\left(T_{e} / m_{e} \Omega_{e}\right)\left(1 / r L_{T_{e}}\right)\right]$, the poloidally asymmetric electron temperature perturbation is given by the steady-state heat balance equation

$$
\vec{\nabla} \cdot \vec{q}_{e}=-Q_{\mathrm{rad}}^{(0)}(r)\left[1+\Delta_{1} \cos \left(\chi-\chi^{*}\right)\right] .
$$

Here $\vec{q}_{e}=-\kappa_{\perp, e} \nabla_{\perp} T_{e}-\kappa_{\|, e} \nabla_{\|} T_{e}$ is the heat flux. Although the perpendicular heat conductivity $\kappa_{\perp, e}$ may be anomalous, the classical parallel electron thermal conductivity $\kappa_{\|, e}$ $=3.2 N_{e} T_{e} / m_{e} \nu_{e}$ plays by far the dominant role if the electron temperature is not uniform on the magnetic surface. ${ }^{15}$ For $\Delta_{1} \sim \mu \ll 1$, the steady-state poloidal variation of the electron temperature due to asymmetric radiation is

$$
t_{e}^{(1)}=-\delta^{(0)} \cos \left(\chi-\chi^{*}\right),
$$

where $\delta^{(0)}(r)=Q_{\mathrm{rad}}^{(0)} \Delta_{1} q^{2} R^{2} / T_{e}^{(0)} \kappa_{\|, e}$ and $t_{e}^{(0)}=t_{e}(\chi) / T_{e}^{(0)}(\psi)$. It is to be noted that the value of $\delta^{(0)}$ is larger at smaller electron temperature. The electron temperature in the edge of all small devices is much smaller than the big devices like Joint European Torus (JET). Thus due to a small edge electron temperature and a smaller parallel conduction of heat (both of which influence the generation of asymmetry in the radiation and the poloidal electron temperature inhomogeneity), the effects are much stronger in smaller devices as compared to big machines like JET. Second, the electron temperature is typically $T_{e}^{(0)} \geqslant 150$ in the edge of the bigger machine and density profile is stepped. This region is stable to ITG mode. Where as the high temperature of electron [i.e., $\left.T_{e}^{(0)} \approx(70-150) e V\right]$ occurs deep inside the edge of smaller devices. The transport in this region is mainly controlled by ITG mode. The flow generated due to asymmetric radiation at higher temperature can stabilize the ITG mode via $\vec{E} \times \vec{B}$ shear rotation. The poloidal asymmetry of the equilibrium electron temperature could also be produced by the Shafranov shift of nonconcentric magnetic surfaces; however, that effect turns out to be quantitatively smaller than the one discussed above.

In the large mobility limit $m_{e} / m_{i} \rightarrow 0$, the poloidal angle $(\chi)$ dependent electron temperature perturbation combines with the leading order parallel electron momentum equation $e N_{e} E_{\|}+\nabla_{\|} P_{e}+0.71 N_{e} \nabla_{\|} T_{e}=0$ to yield the modified electron adiabatic relation

$$
\tau_{i} \phi^{(1)}=n^{(1)}+1.71 t_{e}^{(1)}=n^{(1)}-1.17 \delta^{(0)} \cos \left(\chi-\chi^{*}\right),
$$

where $\tau_{i}=T_{i} / T_{e}$.

The leading order parallel ion momentum equation (i.e., $\left.e N_{i} \nabla_{\|} \phi+\nabla_{\|} N_{i} t_{i}+n T_{i}=0\right)$ yields

$$
\begin{aligned}
t_{i}^{(1)} & =-n^{(1)}-\phi^{(1)} \\
& =-\left(1+1 / \tau_{i}\right) n^{(1)}+1.71\left(\delta^{(0)} / \tau_{i}\right) \cos \left(\chi-\chi^{*}\right) .
\end{aligned}
$$

We next evaluate the toroidal flow perturbation in terms of density and ion temperature perturbations from ion continuity equation. In leading order, the perpendicular ion flow and the continuity Eq. (1) read

$$
\begin{aligned}
& \hat{b} \cdot \vec{U}_{i}=U_{\beta i}=\frac{1}{e B N_{i}} h_{\psi}^{-1}\left(\frac{\partial}{\partial \psi} P_{i}+e N_{i} \frac{\partial \phi}{\partial \psi}\right), \\
& \hat{p} \cdot \vec{U}_{i}=U_{\psi i}=-\frac{1}{e B N_{i}}\left(\frac{B_{\phi}}{B}\right) h_{\chi}^{-1}\left(\frac{\partial}{\partial \chi} P_{i}+e N_{i} \frac{\partial \phi}{\partial \chi}\right),
\end{aligned}
$$

and

$$
\vec{\nabla} \cdot\left(N_{i} \vec{U}_{i}\right)=\frac{1}{J} \frac{\partial}{\partial \chi}\left(h_{\psi} h_{\phi} N_{i} U_{\chi i}\right)=0 .
$$

Combining Eqs. (21) and (22), we obtain

$$
\frac{\partial}{\partial \chi}\left(\frac{N_{i} U_{\| i}}{B}\right)=\left(\frac{h_{\phi} B_{\phi}}{e}\right) \frac{\partial}{\partial \chi}\left[\frac{1}{B^{2}}\left(\frac{\partial P_{i}}{\partial \psi}+e N_{i} \frac{\partial \phi}{\partial \psi}\right)\right]
$$

where the $U_{\chi i}=\left(B_{\phi} / B\right) U_{\beta_{i}}-\left(B_{\chi} / B\right) U_{\| i}$ relation is used. Equation (23) offers the relation between $\partial u_{\| i}^{(1)} / \partial_{\chi}, n^{(1)}$, and $b^{(1)}$. It follows by combining Eqs. (23) and (20)

$$
\begin{aligned}
\frac{\partial u_{\| i}^{(1)}}{\partial \chi}= & -U_{\| i}^{(0)} \frac{\partial}{\partial \chi}\left(n^{(1)}-b^{(1)}\right) \\
& -\frac{T_{i}^{(0)}}{e B} h_{\phi} B_{\phi}\left[\left(\frac{e}{T_{i}^{(0)}} \frac{\partial \phi^{(0)}}{\partial \psi}-\frac{1}{\tau_{i}} \frac{\partial \ln N_{j}^{(0)}}{\partial \psi}\right) \frac{\partial n^{(1)}}{\partial \chi}\right. \\
& -2\left(\frac{e}{T_{i}^{(0)}} \frac{\partial \phi^{(0)}}{\partial \psi}+\left(1+\eta_{i}\right) \frac{\partial \ln N_{i}^{(0)}}{\partial \psi}\right) \frac{\partial b^{(1)}}{\partial \chi} \\
& \left.-\frac{1.71 \delta^{(0)}}{\tau_{i}} \frac{\partial \ln N_{i}^{(0)}}{\partial \psi} \sin \left(\chi-\chi^{*}\right)\right] .
\end{aligned}
$$

We now derive the relation between the density $n^{(1)}$, the temperature $t_{i}^{(1)}$, and magnetic perturbations $b^{(1)}$ from the ion energy equation (3) and obtain 


$$
\begin{aligned}
-\left[\frac{1}{B_{0}} h_{\psi}^{-1} \frac{\partial \phi^{(0)}}{\partial \psi}+\left(\frac{B_{\chi}^{(0)}}{B_{\phi}^{(0)}}\right) U_{\| i}^{(0)}\right] h_{\chi}^{-1} \frac{\partial n^{(1)}}{\partial \chi} \\
-\left[\left(\frac{3}{2} \eta_{i}-1\right)\left(\frac{T_{i}^{(0)}}{e B_{0}}\right) h_{\psi}^{-1} \frac{\partial \ln N^{(0)}}{\partial \psi}\right] \frac{e}{T_{i}^{(0)}} h_{\chi}^{-1} \frac{\partial \phi^{(1)}}{\partial \chi} \\
+\frac{3}{2}\left[\frac{1}{B_{0}} h_{\psi}^{-1} \frac{\partial \phi^{(0)}}{\partial \psi}+\left(\frac{B_{\chi}^{(0)}}{B_{\phi}^{(0)}}\right) U_{\| i}^{(0)}\right] h_{\chi}^{-1} \frac{\partial t_{i}^{(1)}}{\partial \chi}-5\left(\frac{T_{i}^{(0)}}{e B_{0}}\right) \\
\quad \times\left(h_{\psi}^{-1} \frac{\partial \ln T_{i}^{(0)}}{\partial \psi}\right) h_{\chi}^{-1} \frac{\partial b^{(1)}}{\partial \chi}=\chi_{\| i}^{(0)}\left(\frac{B_{\chi}^{2}}{B_{\phi}^{2}}\right) h_{\chi}^{-2} \frac{\partial^{2} t_{i}^{(1)}}{\partial \chi^{2}} .
\end{aligned}
$$

The expressions of $\phi^{(1)}, t_{i}^{(1)}$ from Eqs (21) and (22), and $b^{(1)}=-\varepsilon \cos \chi$ inserting into (25), we obtain the following relation in $n^{(1)}$ :

$$
\begin{aligned}
& {\left[4 U_{E}^{(0)}+\left(\frac{3}{2} \eta_{i}-1\right) U_{* i}^{(0)}+4\left(\frac{B_{\chi}}{B_{\phi}^{(0)}}\right) U_{\| i}^{(0)}\right] \frac{\partial n^{(1)}}{\partial \chi}} \\
& +\frac{3}{2} \times 1.71 \delta^{(0)}\left[U_{E}^{(0)}+\left(\frac{3}{2} \eta_{i}-1\right) U_{* i}^{(0)}\right. \\
& \left.+\left(\frac{B_{\chi}^{(0)}}{B_{(0)}}\right) U_{\| i}^{(0)}\right] \sin \left(\chi-\chi^{*}\right)+5 \varepsilon \eta_{i} U_{* i}^{(0)} \sin \chi \\
& =\frac{r}{q^{2} R^{2}}\left[2 \chi_{\| i}^{(0)} \frac{\partial^{2} n^{(1)}}{\partial \chi^{2}}+1.71 \chi_{\| i}^{(0)} \delta^{(0)} \cos \left(\chi-\chi^{*}\right)\right] .
\end{aligned}
$$

Here $\quad U_{E}^{(0)}=\left(1 / B_{0}\right) h_{\psi}^{-1} \partial \phi^{(0)} / \partial \psi=-E_{r} / B_{0} \quad$ and $\quad U_{*_{i}}^{(0)}$ $=\left(T_{i}^{(0)} / e B_{0}\right) h_{\psi}^{-1} \partial \ln N_{i}^{(0)} / \partial \psi$ are the $\vec{E} \times \vec{B}$ and ion diamagnetic drifts, respectively.

We write the solution of Eq. (26) in the form of

$$
n^{(1)}=\alpha_{1} \cos \chi+\alpha_{2} \sin \chi,
$$

where the coefficients $\alpha_{1}$ and $\alpha_{2}$ are given by

$$
\alpha_{1}=\frac{Q Z-Y S}{Q^{2}+S^{2}} \quad \text { and } \quad \alpha_{2}=-\frac{Q Y+S Z}{Q^{2}+S^{2}}
$$

and

$$
\begin{aligned}
Q= & 4 U_{E}^{(0)}+\left(\frac{3}{2} \eta_{i}-1\right) U_{* i}^{(0)}+4\left(\frac{B_{\theta}}{B_{\phi}}\right) U_{\| i}^{(0)}, \\
S= & 2 \chi_{\| i}\left(\frac{r}{q^{2} R^{2}}\right), \\
Y= & -\frac{3}{2} \times 1.71 \delta^{(0)}\left[U_{E}^{(0)}+\left(\frac{3}{2} \eta_{i}-1\right) U_{* i}^{(0)}\right. \\
& \left.+\left(\frac{B_{\theta}^{(0)}}{B_{(0)}}\right) U_{\| i}^{(0)}\right] \sin \chi^{*}-1.71 \chi_{\| i}^{(0)} \frac{r}{q^{2} R^{2}} \delta^{(0)} \cos \chi^{*}, \\
Z= & 5 \varepsilon \eta_{i} U_{*_{i}}^{(0)}+\frac{3}{2} \times 1.71 \delta^{(0)}\left[U_{E}^{(0)}+\left(\frac{3}{2} \eta_{i}-1\right) U_{*_{i}}^{(0)}\right. \\
& \left.+\left(\frac{B_{\theta}^{(0)}}{B_{(0)}}\right) U_{\| i}^{(0)}\right] \cos \chi^{*}-1.71 \chi_{\| i}^{(0)} \frac{r}{q^{2} R^{2}} \delta^{(0)} \cos \chi^{*} .
\end{aligned}
$$

For large aspect ratio tokamaks with circular cross sections, in which case $\chi=\theta$ and $b^{(1)}=-\varepsilon \cos \theta, \quad h_{\psi}^{-1} \partial / \partial \psi \sim \partial / \partial r$, $\left(B_{\phi} / B\right) h_{\chi}^{-1} \partial / \partial \chi \sim r^{-1} \partial / \partial \theta$, and by substituting the values of $t_{i}^{(1)}, u_{\| i}^{(1)}$, and $n^{(1)}$ as a function of poloidal angle $(\theta)$ from Eqs. (20), (24), and (27) into Eq. (16), we get the equation for the toroidal flow in the presence of asymmetric radiation as

$$
\begin{aligned}
\frac{\partial U_{\phi, i}}{\partial r}+ & \left\{0.2 q^{2} \frac{T_{i}}{e B_{\theta}}\left(\frac{\partial \ln T_{i}}{\partial r}\right)^{2}\right. \\
\times & {\left[1-40.7\left(1+\frac{0.94}{\eta_{i}}+\frac{0.22}{\eta_{i}^{2}}\right) \frac{\delta^{(0)}}{\varepsilon} \cos \theta^{*}\right] } \\
& -4.45\left(\frac{c_{i}^{2} \Omega_{i}}{R^{2} \nu_{i}^{2}}\right) \frac{\delta^{(0)}}{q}\left(\cos \theta^{*}-\sin \theta^{*}\right) \\
& \left.-0.6\left(1-\frac{0.96}{\eta_{i}}\right) \frac{T_{i}}{r e B_{\theta}} \frac{\partial \ln T_{i}}{\partial r} \frac{\Omega_{i}}{\nu_{i}} \delta^{(0)} \varepsilon \sin \theta^{*}\right\}=0,
\end{aligned}
$$

with $Q / S \sim 1.28\left(q^{2} R^{2} \nu_{i} / \Omega_{i} r L_{T_{i}}\right)<1$ and the relation $U_{\theta i}$ $=U_{E}+\left(1+\eta_{i}\right) U_{*_{i}}+\left(B_{\theta} / B_{\phi}\right) U_{\| i}$.

Here the loss of momentum due to charge exchange with neutrals has been ignored in the derivation of Eq. (33). This is acceptable when the neutral density has a value below a critical neutral density. This may be estimated by comparing the momentum loss via the charge exchange term $m_{i} N_{i} \nu_{\mathrm{cx}} U_{\phi, i}$ with $\eta_{2 i} \partial^{2} U_{\phi, i} / \partial r^{2}$ (where $\eta_{2 i}=12 P_{i} \nu_{i} / 10 \Omega_{i}^{2}$ ). This comparison leads to the inequality $N_{0}<3.6 \rho_{i}^{2} \nu_{i} /\left(L_{T}^{2}\right.$ $\left.\langle\sigma v\rangle_{\mathrm{cx}}\right)$ for neglecting the charge exchange effects; here $\nu_{\mathrm{cx}} \cong\left(\langle\sigma v\rangle_{\mathrm{cx}} N_{0}\right) / 3$ is the charge exchange rate, $N_{0}$ is the neutral density, and $\langle\sigma v\rangle_{\mathrm{cx}} \cong 10^{-14} \mathrm{~m}^{3} \mathrm{~s}^{-1}$. Note that in the absence of asymmetric radiation, we recover the results of Refs. 11, 18, 19, and 22. Among the radiation asymmetry driven terms, the second (with the coefficient 4.45) provides the dominant contribution. Going back to the derivation, its origin is a component of the divergence of the Mikhailovsky-Tsypin tensor proportional to $-1.6\left(\chi_{\| i}^{(0)} B_{\chi}^{(0)} / B_{0} h_{\chi}^{(0)}\right) \oint\left(\partial t_{i}^{(1)} / \partial \chi\right)\left(\partial b^{(1)} / \partial \chi\right) d \chi / 2 \pi, \quad$ where $t_{i}^{(1)}=t_{i}(\theta) / T_{i}(r)$ and $b^{(1)}$ is the theta $\theta$ dependent component of the toroidal field. Note that parallel force balance on the electron and ion fluids and the assumption of quasineutrality leads to the relationship $t_{i}^{(1)} \sim t_{e}^{(1)} \sim \delta^{(0)}$, where $\delta^{(0)}$ is related to the radiation asymmetry parameter $\Delta_{1}$. Order of magnitude wise, $\delta^{(0)} \sim Q_{\mathrm{rad}}^{(0)} \Delta_{1} q^{2} R^{2} / T_{e}^{(0)} \kappa_{\|, e}^{(0)} \sim\left(P_{e 0}^{\text {core }} \Delta_{1} / P_{e 0}^{\text {edge }} \nu_{e} \tau_{E}\right)$ $\times\left(q R / \lambda_{f}\right)^{2}$, where $\lambda_{f}$ is the electron mean free path, $\tau_{E}$ is the energy confinement time, and $P_{e 0}^{\text {edge }}$ and $P_{e 0}^{\text {core }}$ are the electron pressure at the edge and at the core, respectively.

\section{APPLICATIONS OF THE THEORY}

We solve Eq. (33) under the local approximation where $\partial_{r} \ln U_{\phi i} \approx \partial_{r} \ln T_{i} \approx-1 / L_{T}$. With these approximations, the steady-state equation for $U_{\phi, i}^{(0)}$ simplifies to 


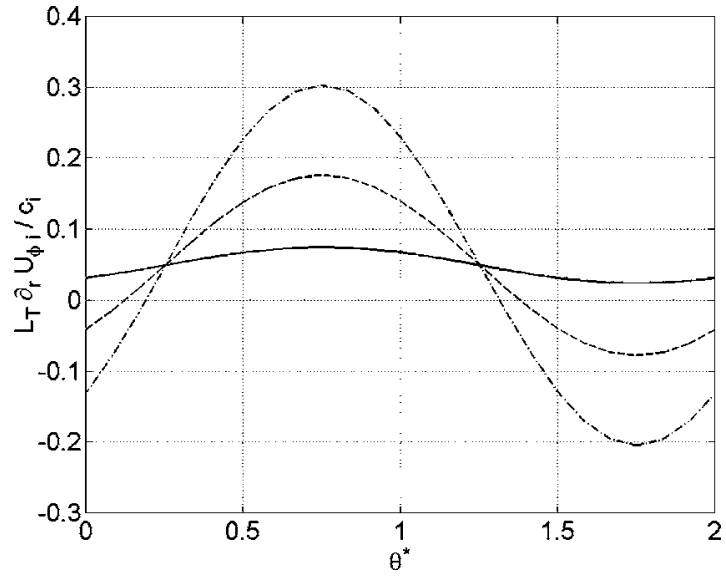

FIG. 1. Plot of the normalized toroidal flow $\left(L_{T_{i}}\left|\partial_{r} U_{\phi, i}\right|\right) / c_{i}$ as a function of $\pi \theta^{*}$ (the poloidal location of maximum radiation due to the seeded impurity) for a fixed value of $Z_{\text {eff }}=1.5$ and various values of the temperature asymmetry parameter $\delta^{(0)}: \delta^{(0)}=1.0 \times 10^{-5}$ (solid line), $\delta^{(0)}=5.0 \times 10^{-5}$ (dasheddotted line), $\delta^{(0)}=1.0 \times 10^{-4}$ (dotted line).

$$
\begin{aligned}
\frac{\partial U_{\phi, i}}{\partial r}= & \left\{-0.8 \frac{q^{3} \varepsilon_{i}^{2}}{\varepsilon} \frac{\rho_{i} c_{i}}{R^{2}}\right. \\
& \times\left[1-40.7\left(1+\frac{0.94}{\eta_{i}}+\frac{0.22}{\eta_{i}^{2}}\right) \frac{\delta^{(0)}}{\varepsilon} \cos \theta^{*}\right] \\
& +4.45\left(\frac{c_{i}^{2} \Omega_{i}}{R^{2} \nu_{i}^{2}}\right) \frac{\delta^{(0)}}{q}\left(\cos \theta^{*}-\sin \theta^{*}\right) \\
& \left.-1.2\left(1-\frac{0.96}{\eta_{i}}\right) \frac{q \varepsilon_{i}}{\varepsilon^{2}} \frac{\rho_{i} c_{i}}{R^{2}} \frac{\Omega_{i}}{\nu_{i}} \delta^{(0)} \varepsilon \sin \theta^{*}\right\} .
\end{aligned}
$$

We now apply our results to TEXTOR-94 RI mode discharges. ${ }^{6-8}$ Edge parameters are typically $T_{i}^{(0)}=150 \mathrm{eV}$, $T_{e}^{(0)}=80 \mathrm{eV}, N_{i}^{(0)}=1 \times 10^{19} \mathrm{~m}^{-3}$, and $L_{T_{i}} \sim 0.05 \mathrm{~m}$ at the radius $r=0.35 \mathrm{~m}$ outside which most of the seeded impurity radiation takes place; moreover, $R=1.75 \mathrm{~m}, a=0.46 \mathrm{~m}$, $B=2.25 \mathrm{~T}, q \approx 3$, and $A_{i}=2$ (atomic mass). In Fig. 1 the normalized toroidal flow $\left(L_{T_{i}}\left|\partial_{r} U_{\phi, i}\right|\right) / c_{i}$ is plotted as a function of the angle $\theta^{*}$ (the location of maximum radiation) for various values of the asymmetry parameter $\left(\delta^{(0)}\right)$ and a fixed value of $Z_{\text {eff }}=1.5$. This figure demonstrates that the toroidal flow may be significantly enhanced by the asymmetric radiation even for values of $\delta^{(0)}$ lower than $0.1 \%$. It is to be noted that the radiation asymmetry and the parallel electron thermal conduction effects determine $\delta^{(0)}$ and is therefore likely to be smaller in bigger, hotter devices like JET, ITER, etc. We may thus conclude that flow generation due to radiation asymmetry effects may not be important for hot edge plasmas.

We now present a semiquantitative explanation of how a small poloidal asymmetry in the radiated power can modify the basic characteristic of L-mode discharges and then lead to a L-RI mode transition. We follow the procedure used by $\operatorname{Tokar}^{8}$ to study the bifurcation of the tokamak plasma; namely, we consider the stationary continuity equation and balance the convective fluxes associated with the various components of the turbulence with the particle sources at the edge.
Particle transport as a function of peaking factor $(p)$ can be described from the steady-state continuity equation

$$
G(p)=\Gamma-r^{-1} \int_{0}^{r} S_{e} r d r, \quad \Gamma=\Gamma_{\mathrm{ITG}}+\Gamma_{\mathrm{DTE}}+D_{\mathrm{DRBM}},
$$

where $\Gamma_{\mathrm{ITG}} \approx-D_{\mathrm{ITG}} \partial_{r} N_{e}, \quad \Gamma_{\mathrm{DTE}} \approx-D_{\mathrm{DTE}}\left(\partial_{r} N_{e}+N_{e} \partial_{r} \ln q\right)$, and $\Gamma_{\mathrm{DRBM}} \approx-D_{\mathrm{DRBM}} \partial_{r} N_{e}$ are the particle fluxes associated with ITG, DTE, and DRBM modes, respectively, and $S_{e}$ is the particle source/unit volume near the edge.

We consider a simple case where the spatial symmetry breaking term due to $\vec{E} \times \vec{B}$ shear rotation reduces the linear growth of the instabilities ${ }^{12}$ and modifies the steady-state condition in the continuity equation to yield a new expression for the peaking factor $p=1 / \eta_{i}{ }^{8}$ In the presence of $\vec{E} \times \vec{B}$ shear rotation, the reduced linear growth rate of the background instabilities has the general form $\gamma=\gamma_{\mathrm{ln}}\left(1-\Omega^{2}\right)$, where $\gamma_{1 \mathrm{n}}$ is the linear growth rate and $\gamma_{1 \mathrm{n}} \Omega^{2}$ is the shear damping rate; $\Omega \equiv k_{\theta} U_{E}^{\prime} W_{k} / \gamma_{\mathrm{ln}}$ is the average radial symmetry breaking term, with $k_{\theta}$ the poloidal wave vector and $W_{k}$ the radial width of the unstable mode. ${ }^{12}$ As stated earlier we assume that the ITG, DTE and high- $m$ DRB modes dominate the transport of ionized particles at the plasma edge. The linear growth rates of these modes are well known and may be described as follows:

(a) The ITG growth rate ${ }^{13}$

$$
\gamma^{\mathrm{ITG}}=\gamma_{\ln }^{\mathrm{ITG}}\left(1-\Omega^{2}\right),
$$

where $\quad \gamma_{\ln }^{\mathrm{ITG}} \cong \gamma_{0}^{\mathrm{ITG}} F^{\mathrm{ITG}}(p)$, $=\sqrt{\left(1-0.17 p-0.25 \varepsilon_{i} p^{2}-1.36 / \varepsilon_{i}\right)}$, $=2\left(k_{\theta} \rho_{s} c_{s} / R\right) \varepsilon_{i}^{1 / 2}, k_{\theta} \rho_{s} \approx 0.05$.

and $\underset{\gamma_{0}^{\mathrm{ITG}}(p)}{\gamma_{\mathrm{ITG}}}$

(b) The DTE mode growth rate ${ }^{8}$

$$
\gamma_{\ln }^{\mathrm{DTE}} \cong \gamma_{0}^{\mathrm{DTE}} F^{\mathrm{DTE}}(p)
$$

where $F^{\mathrm{DTE}}=p$ and $\gamma_{0}^{\mathrm{DTE}}=8\left(k_{\theta} \rho_{s} c_{s} / R\right)^{2}\left(r \varepsilon_{e} \varepsilon_{i} / R \nu_{e}\right) f_{t r}$.

(c) The linear growth rate of the high- $m$ DRB mode

$$
\gamma_{\mathrm{ln}}^{\mathrm{DRBM}} \approx c_{s}\left(\sqrt{2 / R L_{n}}\right)
$$

$\gamma_{\mathrm{ln}}^{\mathrm{DRBM}}=\gamma_{0}^{\mathrm{DRB}} F^{\mathrm{DRB}}(p), \gamma_{0}^{\mathrm{DRB}}=2 c_{s} / R \varepsilon_{i}^{1 / 2}, F^{\mathrm{DRB}}(p)=p^{1 / 2}$, and $k_{r}^{-1} \approx\left(2 \pi q \rho_{s}\right)^{2}\left(R \nu_{e} / c_{e}\right)\left(m_{e} / m_{i}\right)^{1 / 2} \varepsilon_{i}^{1 / 2} p^{1 / 2}$ is the radial correlation length. ${ }^{14}$

Particle transport due to these instabilities is estimated from the mixing length argument, which yields $D^{A N}$ $\approx \gamma^{\ln } / k_{r}^{2}$. The equation for the peaking factor that finally emerges is 


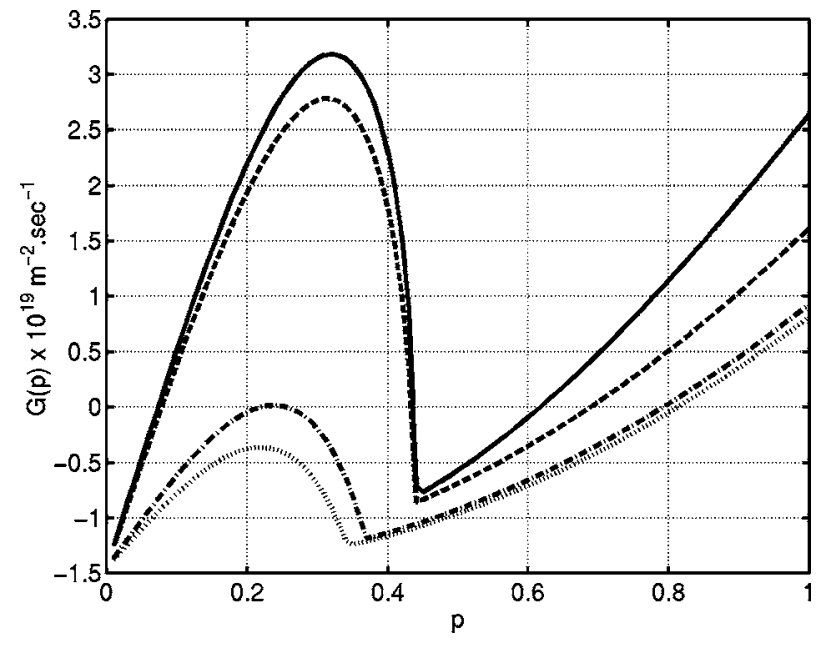

FIG. 2. The turbulent particle flux associated with ITG, DTE, and DRB modes as a function of the peaking parameter $p=1 / \eta_{i}$ for $\delta^{(0)}=1.0 \times 10^{-5}$ (solid line), $\delta^{(0)}=1.0 \times 10^{-4}$ (dashed), $\delta^{(0)}=3.7 \times 10^{-4}$ (dashed-dotted), and $\delta^{(0)}=4.0 \times 10^{-4}($ dotted $)$.

$$
\begin{aligned}
G(p)= & \Gamma_{0}\left[\frac{p}{\varepsilon_{i}^{1 / 2}}\left(F(p)-\frac{\gamma_{E \times B}}{\gamma_{0}^{\mathrm{TG}}}\right)\right. \\
& +2\left(\frac{r}{R}\right)^{3 / 2} \frac{\varepsilon_{e} c_{s}}{\nu_{e} R}\left(p-0.5 \frac{\hat{s}}{\varepsilon_{i} \varepsilon}\right)\left(p-\frac{\gamma_{E \times B}}{\gamma_{0}^{\mathrm{DTE}}}\right) \\
& \left.+2(\pi q)^{2}\left(\frac{m_{e}}{m_{i}}\right)^{1 / 2}\left(\frac{R \nu_{e}}{c_{e}}\right) p^{3 / 2}\left(p^{1 / 2}-\frac{\gamma_{E \times B}}{\gamma_{0}^{\mathrm{DRBM}}}\right)\right]-\hat{S}=0 .
\end{aligned}
$$

Here the three first terms represent the particle fluxes related to the ITG, DTE, and DRB mode instabilities, including the reduction factor associated with $\vec{E} \times \vec{B}$ shear $\left[\propto \gamma^{\ln } \Omega^{2}\right.$ $\left.\equiv \gamma_{E \times B} \approx\left(\left\langle U_{E}\right\rangle^{\prime}\right)^{2} / \gamma^{\ln }\right]$ and particle pinch associated with the DTE mode $\left(\propto 0.5 \hat{s} / \varepsilon_{i} \varepsilon\right)$; the last term represents the inward particle flux originating from the edge; $\hat{S}=r^{-1} \int_{0}^{r} S_{e} r d r$, $\sim 2.5 \times 10^{19} \mathrm{~m}^{-2} \mathrm{~s}^{-1}{ }^{8}, \Gamma_{0}=8 N_{e} c_{s}\left(\rho_{s} \varepsilon_{i} / R\right)^{2}$, and $k_{\theta} \rho_{s} \sim 0.5$ are assumed throughout. If $\vec{E} \times \vec{B}$ shear stabilization is strong enough to completely stabilize any of the instabilities, the corresponding contribution to the particle flux is taken as zero.

Figure 2 shows a plot of $G(p)$ for a fixed value of $Z_{\text {eff }}=1.5$ and various values of the radiation asymmetry parameter $\Delta_{1}$. It is noted that for low values of $\Delta_{1}, G(p)$ vanishes for three values of $p$, including a low one; thus the discharge can settle in a stable stationary state with a low peaking factor and stay there without any bifurcation. This is because we have chosen a value of $Z_{\text {eff }}$ that is lower than that considered by Tokar $^{8}$ so that the bifurcations studied by him are not operative. Even at these lower values of $Z_{\text {eff }}$, the plasma can, however, display bifurcations into a peaked density state when $\Delta_{1}$ exceeds about $9 \%$; in these cases the equation $G(p)=0$ has only one real root, which corresponds to a relatively high peaking factor. The reason for the density peakedness is the considerable increase of the velocity shear at higher values of $\Delta_{1}$; that reduces the growth rates [e.g., $F(p)-\gamma_{E \times B} / \gamma_{0}$ ] at constant $p$ and hence requires higher values of peaking parameter to balance the particle source.

\section{CONCLUSIONS}

In conclusion, we have shown that small poloidal asymmetries in the radiation can lead to significant sheared neoclassical toroidal flows in an impurity seeded tokamak plasma. We have shown that these flows contribute to the stabilization of edge instabilities and lead to the bifurcation of the plasma into an improved confinement mode, viz., the RI mode. Such effects are likely to be important only for tokamak plasmas with significant collisionality in the edge region. The transition triggered by poloidal asymmetries takes place at lower plasma dilution factors than that triggered by the reduction of the ITG growth rate at large value of $Z_{\text {eff }}$. We have carried out the calculations in the collisional neoclassical limit; they are applicable to the outer $25 \%$ of the plasma where radiative effects are large. However, the sheared flow may extend to the plasma interior, either through various possible momentum pinch mechanisms (see, e.g., Ref. 20) or through inward propagation of a front owing to increased variation of $\partial T_{i} / \partial r$ and $\partial E_{r} / \partial r$ (see Sec. 5.3 of Ref. 21). We have neglected the anomalous toroidal momentum relaxation for the strongly collisional edge in the present analysis. The anomalous radial viscosity can contribute to momentum relaxation but it is not necessary that the steadystate radial electric field has anomalous feature. Even if it is so; we believe that there must be some contribution from neoclassical effects on steady-state toroidal flow and the electric field. In the our paper, we derive the expressions of neoclassical radial electric and toroidal flows that can be compared to the experimental results in a collisional edge plasma to pin down whether the neoclassical radial electric field and toroidal flows have neoclassical or anomalous characteristics. $^{22}$

\section{APPENDIX: THE STRESS TENSORS $\left(\stackrel{\pi}{\pi}_{I}\right)$}

In the limit $\nu_{i} / \Omega_{i} \ll 1$, the stress tensor $^{15}$ with the Mikhailovskii and Tsypin ${ }^{16}$ correction can be split into three parts:

$$
\overleftrightarrow{\pi}_{i}=\overleftrightarrow{\pi}_{0, i}+\overleftrightarrow{\pi}_{3-4, i}+\overleftrightarrow{\pi}_{1-2, i}
$$

The parallel stress tensor or diagonal matrix $\left(\overleftrightarrow{\pi}_{0 i}\right)$, the gyrostress tensor $\left(\stackrel{\leftrightarrow}{\pi}_{3-4,}\right)$, and the perpendicular stress tensor $\left(\stackrel{\pi}{1-2, i}_{1}\right)$ are given as follows. ${ }^{16,17}$ The parallel stress tensor is 


$$
\begin{aligned}
\overleftrightarrow{\pi}_{0 i}= & -3 \eta_{0 i}\left(\hat{n} \hat{n}-\frac{\overleftrightarrow{I}}{3}\right)\left\{\hat{n} \cdot \vec{\nabla} \vec{U}_{i} \cdot \hat{n}-\frac{\vec{\nabla} \cdot \vec{U}_{i}}{3}+1.615 \times \frac{2}{5 P_{i}}\left(\hat{n} \cdot \vec{\nabla} \vec{q}_{i} \cdot \hat{n}-\frac{\vec{\nabla} \cdot \vec{q}_{i}}{3}\right)+0.615\right. \\
& \left.\times \frac{2}{5 P_{i}} \times\left[-\left(\hat{n} \cdot \vec{\nabla} \vec{q}_{i}^{*} \cdot \hat{n}-\frac{\vec{\nabla} \cdot \vec{q}_{i}^{*}}{3}\right)+\frac{\vec{q}_{i} \cdot \vec{\nabla} \ln P_{i}}{3}-\frac{\left(2 \vec{q}_{i}-\vec{q}_{i}^{*}\right) \cdot \vec{\nabla} \ln T_{i}}{3}\right]\right\} .
\end{aligned}
$$

The gyrostress tensor is

$$
\begin{aligned}
\overleftrightarrow{\pi}_{3-4, i}= & -\eta_{3, i}\left\{(\hat{p} \hat{p}-\hat{b} \hat{b})\left[\hat{b} \cdot \vec{\nabla} \vec{U}_{i} \cdot \hat{p}+\hat{p} \cdot \vec{\nabla} \vec{U}_{i} \cdot \hat{b}+\frac{2}{5 P_{i}}\left(\hat{b} \cdot \vec{\nabla} \vec{q}_{i} \cdot \hat{p}+\hat{p} \cdot \vec{\nabla} \vec{q}_{i} \cdot \hat{b}\right)\right]-(\hat{p} \hat{b}+\hat{b} \hat{p})\right. \\
& \times\left[\hat{p} \cdot \vec{\nabla} \vec{U}_{i} \cdot \hat{p}-\hat{b} \cdot \vec{\nabla} \vec{U}_{i} \cdot \hat{b}+\frac{2}{5 P_{i}}\left(\hat{p} \cdot \vec{\nabla} \vec{q}_{i} \cdot \hat{p}-\hat{b} \cdot \vec{\nabla} \vec{q}_{i} \cdot \hat{b}\right)\right]+2(\hat{p} \hat{n}+\hat{n} \hat{p}) \\
& \times\left[\hat{b} \cdot \vec{\nabla} \vec{U}_{i} \cdot \hat{n}+\hat{n} \cdot \vec{\nabla} \vec{U}_{i} \cdot \hat{b}+\frac{2}{5 P_{i}}\left(\hat{b} \cdot \vec{\nabla} \vec{q}_{i} \cdot \hat{n}+\hat{n} \cdot \vec{\nabla} \vec{q}_{i} \cdot \hat{b}\right)\right]-2(\hat{b} \hat{n}+\hat{n} \hat{b}) \\
& \left.\times\left[\hat{p} \cdot \vec{\nabla} \vec{U}_{i} \cdot \hat{n}+\hat{n} \cdot \vec{\nabla} \vec{U}_{i} \cdot \hat{p}+\frac{2}{5 P_{i}}\left(\hat{p} \cdot \vec{\nabla} \vec{q}_{i} \cdot \hat{n}+\hat{n} \cdot \vec{\nabla} \vec{q}_{i} \cdot \hat{p}\right)\right]\right\}
\end{aligned}
$$

The perpendicular stress tensor

$$
\begin{aligned}
\pi_{1-2, i}= & -\eta_{1, i}\left\{(\hat{p} \hat{p}-\hat{b} \hat{b})\left[\hat{p} \cdot \vec{\nabla} \vec{U}_{i} \cdot \hat{p}-\hat{b} \cdot \vec{\nabla} \vec{U}_{i} \cdot \hat{b}+\frac{2}{5 P_{i}}\left(\hat{p} \cdot \vec{\nabla} \vec{q}_{i} \cdot \hat{p}-\hat{b} \cdot \vec{\nabla} \vec{q}_{i} \cdot \hat{b}\right)\right]+(\hat{p} \hat{b}+\hat{b} \hat{p})\right. \\
& \times\left[\hat{p} \cdot \vec{\nabla} \vec{U}_{i} \cdot \hat{b}+\hat{b} \cdot \vec{\nabla} \vec{U}_{i} \cdot \hat{p}+\frac{2}{5 P_{i}}\left(\hat{p} \cdot \vec{\nabla} \vec{q}_{i} \cdot \hat{b}+\hat{b} \cdot \vec{\nabla} \vec{q}_{i} \cdot \hat{p}\right)\right]+4(\hat{p} \hat{n}+\hat{n} \hat{p}) \\
& \times\left[\hat{p} \cdot \vec{\nabla} \vec{U}_{i} \cdot \hat{n}+\hat{n} \cdot \vec{\nabla} \vec{U}_{i} \cdot \hat{p}+\frac{2}{5 P_{i}}\left(\hat{p} \cdot \vec{\nabla} \vec{q}_{i} \cdot \hat{n}+\hat{n} \cdot \vec{\nabla} \vec{q}_{i} \cdot \hat{p}\right)\right]+4(\hat{b} \hat{n}+\hat{n} \hat{b}) \\
& \left.\times\left[\hat{b} \cdot \vec{\nabla} \vec{U}_{i} \cdot \hat{n}+\hat{n} \cdot \vec{\nabla} \vec{U}_{i} \cdot \hat{b}+\frac{2}{5 P_{i}}\left(\hat{b} \cdot \vec{\nabla} \vec{q}_{i} \cdot \hat{n}+\hat{n} \cdot \vec{\nabla} \vec{q}_{i} \cdot \hat{b}\right)\right]\right\},
\end{aligned}
$$

where $\quad \eta_{0, i}=0.96 P_{i} \nu_{i}^{-1}, \quad \vec{q}_{i}=-P_{i} / m_{i}\left[3.9 / \nu_{i} \hat{n} \hat{n} \cdot \vec{\nabla} T_{i}-5 / 2 \Omega_{i} \hat{n}\right.$ $\left.\times \vec{\nabla} T_{i}-2 \nu_{i} / \Omega_{i}^{2} \hat{n} \times\left(\hat{n} \times \vec{\nabla} T_{i}\right)\right]$ the heat fluxes, $\vec{q}_{i}^{*}$ $=1.04 P_{i} / m_{i} \nu_{i} \hat{n} \hat{n} \cdot \vec{\nabla} T_{i}$, the indexes 3-4 and 1-2 refer to Braginskii's coefficients ${ }^{15} \quad \eta_{3, i}=P_{i} / \Omega_{i}, \quad \eta_{1 i}=3 P_{i} \nu_{i} / 10 \Omega_{i}^{2}$, $\eta_{2 i}=4 \eta_{1 i}$, and $\eta_{4 i}=2 \eta_{3 i}$.

${ }^{1}$ A. Rogister, Phys. Rev. Lett. 81, 3663 (1998); A. Rogister, J. E. Rice, A. Nicolai, A. Ince-Cushman, S. Gangadhara, and Alcator C-Mode Group, Nucl. Fusion 42, 1144 (2002), and references therein; M. Tokar, R. Jaspers, H. R. Koslowski, A Kramer-Flecken, A. M. Messiaen, J. Ongena, A. Rogister, B. Unterberg, and R. R. Weynants, Plasma Phys. Controlled Fusion 41, B317 (1999).

${ }^{2}$ J. E. Rice, P. T. Bonoli, J. A. Goetz, M. J. Greenwald, I. H. Hutchinson, E. S. Marmar, M. Porkolab, S. M. Wolfe, S. J. Wukitch, and C. S. Chang, Nucl. Fusion 39, 1175 (1999).

${ }^{3}$ C. S. Chang, C. K. Phillips, R. White, S. Zweben, P. T. Bonoli, J. E. Rice, M. J. Greenwald, and J. deGrassie, Phys. Plasmas 6, 1969 (1999).

${ }^{4}$ T. Fulop, P. Helander, and P. J. Catto, Phys. Rev. Lett. 89, 225003 (2002).

${ }^{5}$ A. Lazarus, J. D. Bell, C. E. Bush et al., J. Nucl. Mater. 121, 61 (1984).

${ }^{6}$ A. M. Messiaen, J. Ongena, U. Samm et al., Phys. Rev. Lett. 77, 2487 (1996).

${ }^{7}$ R. Weynants, A. Messiaen, J. Ongena et al., Nucl. Fusion 39, 1637 (1999).

${ }^{8}$ M. Z. Tokar, J. Ongena, B. Unterberg, and R. R. Weynants, Phys. Rev. Lett. 84, 895 (2000).
${ }^{9}$ D. R. Baker and M. N. Rosenbluth, Phys. Plasmas 5, 2936 (1998).

${ }^{10}$ M. Z. Tokar, R. Jaspers, R. R. Weynants, H. R. Koslowski, A. KrämerFlecken, A. M. Messiaen, J. Ongena, and B. Unterberg, Plasma Phys. Controlled Fusion 41, L9 (1999).

${ }^{11}$ H. A. Claassen, H. Gerhauser, A. Rogister, and C. Yarim, Phys. Plasmas 7, 3699 (2000).

${ }^{12}$ B. A. Carreras, D. Newman, P. H. Diamond, and Y.-M. Liang, Phys. Plasmas 1, 4014 (1994); T. S. Hahm and K. H. Burrel, Phys. Plasmas 2, 1648 (1995).

${ }^{13} \mathrm{~J}$. Weiland, Collective Modes in Inhomogeneous Plasma (IOP, London, 2000), p. 132.

${ }^{14}$ S. V. Novakovskii, P. N. Guzdar, J. F. Drake, C. S. Liu, and F. L. Waelbroeck, Phys. Plasmas 2, 781 (1995); P. N. Guzdar, J. F. Drake, D. McCarthy, A. B. Hassam, and C. S. Liu, Phys. Fluids B 5, 3712 (1993); B. N. Rogers, J. F. Drake, and A. Zeiler, Phys. Rev. Lett. 81, 4396 (1998).

${ }^{15}$ S. I. Braginskii, in Reviews of Plasma Physics, edited by M. A. Leontovich (Consultants Bureau, New York, 1965), Vol. 1, P. 214.

${ }^{16}$ A. B. Mikhailovskii and V. S. Tsypin, JETP 56, 75 (1982); A. B. Mikhailovskii and V. S. Tsypin, Beitr. Plasmaphys. 24, 335 (1984).

${ }^{17}$ A. Rogister, Phys. Plasmas 1, 619 (1994).

${ }^{18}$ H. A. Claassen, H. Gerhauser, A. Rogister, and C. Yarim, Phys. Plasmas 7, 3699 (2000).

${ }^{19}$ R. Singh, A. Rogister, and P. K. Kaw, Phys. Plasmas 11, 129 (2004).

${ }^{20}$ B. A. Carreras, D. Newman, P. H. Diamond, and Y.-M. Liang, Phys. Plasmas 1, 4014 (1994).

${ }^{21}$ K. C. Shaing, Phys. Rev. Lett. 86, 640 (2001).

${ }^{22}$ P. J. Catto and A. N. Simakov, Phys. Plasmas 12, 012501 (2005). 